\title{
Differential Gene Expression of Pancreatic Cancer Cell Line Capan-2 in Response to Gemcitabine plus BVDU (RP101) Combinatorial Treatment
}

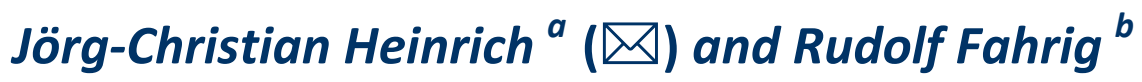 \\ a Technische Universität Dresden, Biotechnology Center, Bioinformatics Group, Tatzberg 47/49, \\ 01307 Dresden, Germany, http://www.biotec.tu-dresden.de \\ b RESprotect GmbH - Prevention of Chemoresistance, Krippener Str. 18b, 01259 Dresden, Germany \\ http://www.resprotect.de
}

\begin{abstract}
ARTICLE INFO:
RECEIVED: 28 July 2015

REVISED: 12 Sep 2015

ACCEPTED: 28 Sep 2015

ONLINE: 07 Oct 2015
\end{abstract}

KEYWORDS:

cancer chemotherapy

Prevention of

chemoresistance

HSP27

HSPB1

BVDU (RP101)

pancreatic adenocarcinoma

\section{A B STRACT}

Every year worldwide approximately 3 million cancer patients receive chemotherapy. Unfortunately in many cases the treatment does not prolong patient's survival time. Chemotherapy and radiation therapy still remain the major strategies with or without surgery. One of the main obstacles in chemo- and radiation therapy is the rapid development of resistance by the cancer cells.

In recent years, the Heat Shock Protein HSP27 has been identified as one of the key players driving resistance development. HSP27 is overexpressed in many kinds of cancer and influences cellular processes like apoptosis (programmed cell death), DNA repair, recombination, and metastasis. As a result, cancer cells are able to suppress apoptosis and develop resistance towards cytostatic drugs such as Gemcitabine which is used as standard therapy in pancreatic cancer.

To address this problem the HSP27-inhibitor BVDU ((E)-5-(2-Bromoviny/)-2'-deoxyuridine, RP101) was administered in combination with Gemcitabine to prevent resistance development. In the study design, the human pancreatic adenocarcinoma cell line Capan- 2 was exposed to Gemcitabine alone and in combination with BVDU. Differential gene expression was displayed using DNA microarrays.

This paper presents the resulting dataset, with pairwise comparison of control (untreated), Gemcitabine-, BVDU-, and Gemcitabine+BVDU treated probes. The five spreadsheet files are openly accessible for validation, re-use and aggregation in future research. 


\section{Overview}

One of the main obstacles in cancer chemotherapy is the rapid development of resistance by cancer cells. In recent years it has been found that the Heat Shock Protein HSP27 is one of the key players driving resistance development. HSP27 is overexpressed in many kinds of cancer and influences cellular processes like apoptosis (programmed cell death), DNA repair, recombination, and metastasis. As a result, cancer cells evade apoptosis and develop resistance towards cytostatic drugs. ${ }^{1-3}$

To address this problem the HSP27-inhibitor BVDU ((E)-5-(2-Bromovinyl)-2'-deoxyuridine, RP101) was administered in combination with Gemcitabine to prevent resistance development.

The human pancreatic adenocarcinoma cell line Capan-2 was exposed to the cytostatic drug Gemcitabine-which is used as standard therapy in pancreatic cancer-in combination with or without BVDU and differential gene expression was displayed using DNA microarrays.

\section{Method}

\section{BVDU co-treatment of Capan-2 cells}

Logarithmically growing cells were seeded at a density of 100,000 cells $/ \mathrm{ml}$ and incubated with 2 $\mathrm{ng} / \mathrm{ml}$ Gemcitabine. Cells were grown to 80 percent confluence and serially passaged. The dose of Gemcitabine was increased with each passage:

- first passage $2 \mathrm{ng} / \mathrm{ml}$ Gemcitabine

- second passage $5 \mathrm{ng} / \mathrm{ml}$ Gemcitabine

- third passage $8 \mathrm{ng} / \mathrm{ml}$ Gemcitabine.

In parallel, cells were treated with Gemcitabine plus $30 \mu \mathrm{M}$ BVDU. The dose of BVDU remained unchanged throughout the experiment. Untreated cells and BVDU-only treatment served as controls. The control group treated with $30 \mu \mathrm{M}$ BVDU only grew as fast as untreated cells, whereas cells treated with Gemcitabine plus BVDU grew significantly more slowly during the third passage compared to cells treated with Gemcitabine only.

RNA probes were extracted when the Gemcitabine-only group reached 80 percent confluence in the third passage. The following total RNA samples were prepared:

1. Control (untreated)

2. BVDU

3. Gemcitabine

4. Gemcitabine + BVDU.
Total RNA was prepared using the QIAGEN RNEasy kit following the manufacturer's instructions (including the DNAse digestion step). Total RNA samples were snap-frozen in liquid nitrogen at a concentration of $0.5 \mu \mathrm{g} / \mu \mathrm{l}$.

\section{Data processing}

Samples were sent to the Affymetrix Screening Service at RZPD (Deutsches Ressourcenzentrum für Genomforschung, Berlin, Germany) for probe labelling, and hybridization to Affymetrix DNA microarrays. The Microarray Suite (MAS) 4.0 software was used for data processing.

\section{Data Description}

Microarrays were compared pairwise. Example: chip A (experiment) was compared with chip B (baseline): chip A versus chip B. The dataset presented comprises the following comparisons:

- BVDU versus control (B2 vs K2),

- Gemcitabine versus control (G_neu2_vs_K2)

- Gemcitabine + BVDU versus control (G+B_2_vs_K2)

- Gemcitabine + BVDU versus Gemcitabine (G+B_2_vs_G_neu2)

- Gemcitabine + BVDU versus BVDU (G+B_2_vs_B2).

Each table is structured in the following columns:

A: Affimetrix internal gene name

B: Stat Pairs: The number of probe pairs in the probe set

C: Stat Pairs Used: The number of probe pairs in the probe set used in the Detection call

D: Signal: A measure of the relative abundance of a transcript

E: Detection: Present (P), Marginal (M) or Absent (A)

F: Detection $p$-value: value which tells in dependence of $R$ and Tau (see there) the significance of the detection value (the lower the more significant the value)

G: Stat Common Pairs: The number of common probe pairs in two arrays (experiment versus baseline) after saturation across the probe set is determined

H: Signal Log Ratio: The change in expression level for a transcript between a baseline and an experiment array. This change is expressed as the $\log 2$ ratio. (A $\log 2$ ratio of 1 is a Fold Change of 2; a log2 ratio of 0.585 is a Fold Change of 1.5) 
I: Signal Log Ratio Low: The lower limit of the Signal Log Ratio within a 95 percent confidence interval.

J: Signal Log Ratio High: The upper limit of the Signal Log Ratio within a 95 percent confidence interval.

K: Change: A call indicating an Increase (I), Marginal Increase (MI), No Change (NC), Decrease (D), or Marginal Decrease (MD) in transcript level between a baseline array and an experiment array.

$\mathrm{L}$ : Change $\mathrm{p}$-value: The $\mathrm{p}$-value ranges in scale from 0.0 to 1.0 , and provides a measure of the likelihood of change and direction. Values close to 0.0 indicate likelihood for an increase in transcript expression level in the experiment array compared to the baseline, whereas values close to 1.0 indicate likelihood for a decrease in transcript expression level. Values near 0.5 indicate a weak likelihood for change in either direction. Hence, the p-value scale is used to generate discrete change calls using thresholds.

\section{Discussion}

Cancer cells develop drug resistance rapidly when exposed to cytostatics. HSP27 inhibition represents a promising approach to counteract this problem. In this study the HSP27 inhibiting small molecule BVDU (RP101) was tested for prevention of Gemcitabine-resistance development. The pancreatic cancer cell line Capan-2 grew significantly slower under combinatorial treatment compared to cells treated with Gemcitabine only. The BVDUonly group showed the same growth characteristics as untreated controls. RNA samples were extracted after 30 days of treatment (third passage) when differential growth between the Gemcitabine plus BVDU group and the Gemcitabine-only group was observed.

Expression profiles reflecting this situation might be advantageous as the base to create computational models predicting resistance development against cancer chemotherapy.

\section{Data Files}

The experimental data described in this paper is presented in five openly accessible spreadsheets. These are:

- BVDU treated versus control (untreated) probes: "01201_1_BVDU_vs_control.xIs"

- Gemcitabine treated versus control probes: "01201_2_Gemcitabine_vs_control.xls"

- Gemcitabine + BVDU treated versus control probes:

"01201_3_Gemcitabine+BVDU_vs_control.xIs"

- Gemcitabine + BVDU treated versus Gemcitabine treated probes:

"01201_4_Gemcitabine+BVDU_vs_Gemcitabine.xIs"

- Gemcitabine + BVDU treated versus BVDU treated probes:

"01201_5_Gemcitabine+BVDU_vs_BVDU.x/s"

All five files are available also in the Open Data Spreadsheet format.

\section{References}

${ }^{1}$ Heinrich JC, Tuukkanen A, Schroeder M, Fahrig $\mathrm{T}$, and Fahrig R. BVDU (Brivudine) binds to heat shock protein HSP27 (HSP27) and enhances survival in animals and pancreatic cancer patients. Journal of Cancer Research and Clinical Oncology. 2011 Sep; 137(9): 1349-61. DOI: 10.1007/s00432011-1005-1.

${ }^{2}$ Fahrig R, Quietzsch D, Heinrich JC, Heinemann V, Boeck S, Schmid RM, Praha C, Liebert A, Sonntag D, Krupitza G, Hänel M. BVDU improves the efficacy of chemotherapy in pancreas carcinoma cell lines and pancreatic cancer patients. Anticancer Drugs. 2006 Oct;17(9):104556. DOI: 10.1097/01.cad.0000231472.92406.d2. PMID: 17001178.

${ }^{3}$ Fahrig R, Heinrich JC, Nickel B, Wilfert F, Leisser C, Krupitza G, Praha C, Sonntag D, Fiedler B, Scherthan $H$, Ernst $H$. Inhibition of induced chemoresistance by cotreatment with (E)-5-(2bromovinyl)-2'-deoxyuridine (BVDU). Cancer Res. 2003 Sep 15;63(18):5745-53. PMID: 14522895. 